Sicherheit des Betriebes von Wichtigkeit, daß an dem Meßort ein zuverlässiger Schutz gegen Kurzschluß, zu hohe Berührungsspannung und zu hohe Temperatur gewährleistet ist.

Von Brown, Boveri \& Cie. AG - Werk Stotz-Kontakt - ist nun eine druckfeste Ex-Kombination entwickelt worden, in die ein Fehlerstrom- und ein Leitungs-Schutzschalter eingebaut sind. Dadurch werden Kurzschluß- und Überlastungsschutz sowie der Schutz gegen zu hohe Berührungsspannung gesichert. Über die Spule des Fehlerstrom -Schutzschalters ist außerdem noch der innere Mantel eines doppelt voneinander isolierten Bleimantelkabels geführt. Bei zu hohen Temperaturen ab etwa $200^{\circ} \mathrm{C}$ schmilzt die Isolation zwischen den zwei Bleimänteln, und der Fehlerstromschutzschalter spricht an. Nach dem Auslösen des Automaten oder des Fehlerstrom-Schutzschalters kann die ExKombination durch den Trennschalter ohne Öffnen des druckfesten Raumes wieder eingeschaltet werden.

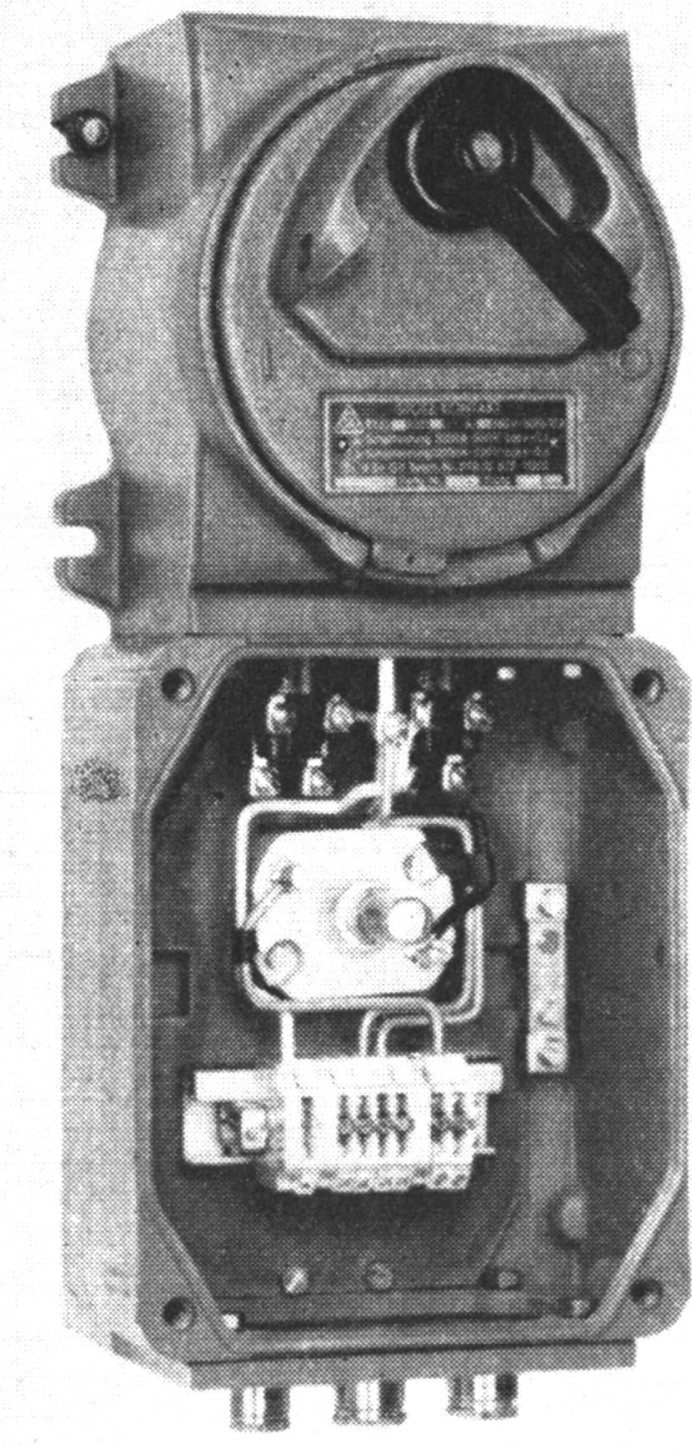

Abb. 1. Ex-Kombination EXKO 6252 D ohne Anschlußkastendeckel

(Werkbild: Brown Boveri)

\section{Elektrothermische Kühlgeräte}

Die Firma $D r$. Neumann Meß- und Regeltechnik München hat neue Kühlgeräte entwickelt, die auf dem Peltiereffekt beruhen und für Labor- und Technikumsversuche bei niederen Temperaturen eine wertvolle Hilfe bilden.

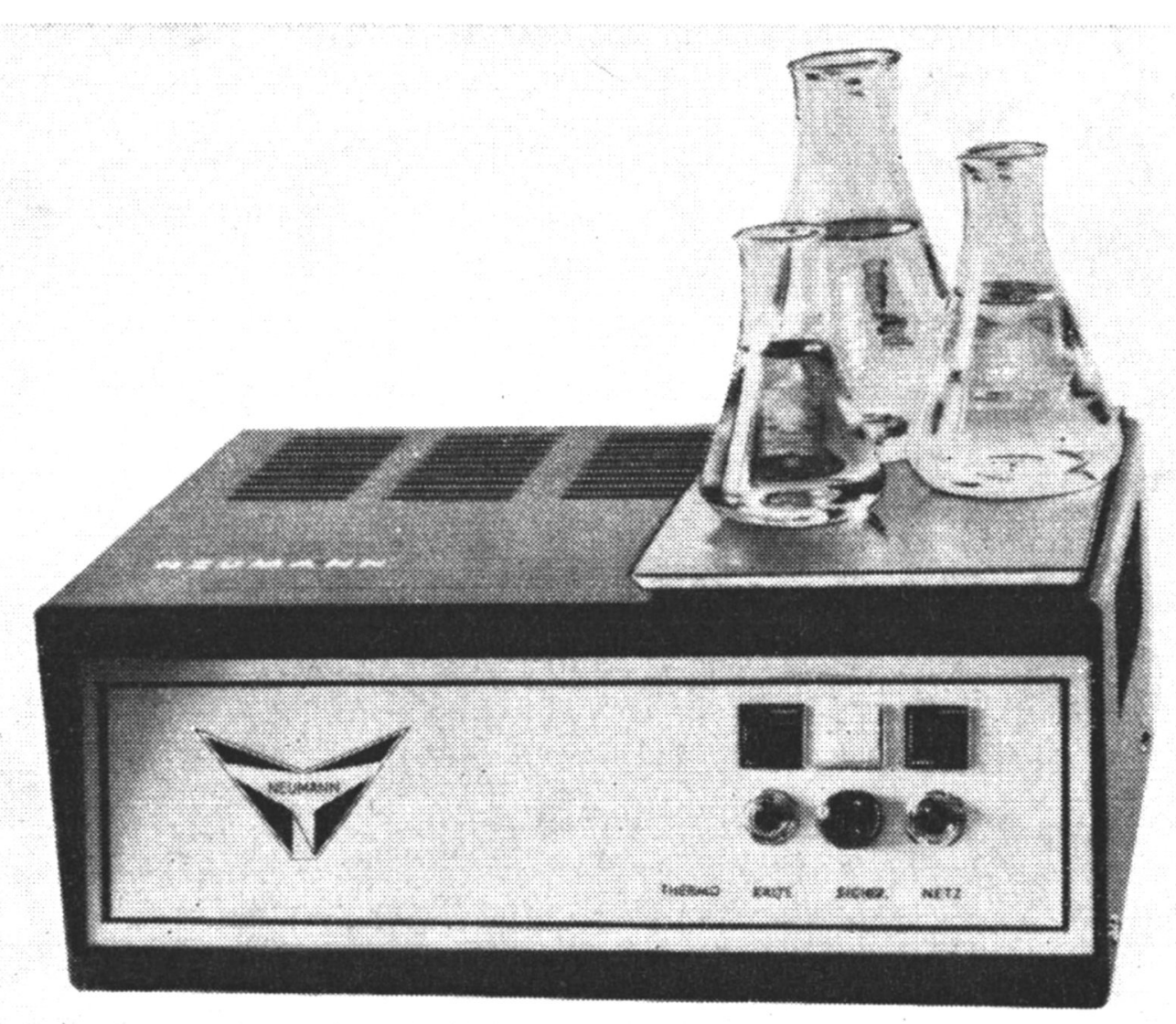

Abb. 2. Neumann-peitogat I - Type WB

(Werkbild: Dr. Neumann Meß- und Regeltechnik)

Die einfachen und verschleißfreien Peltierbatterien sind in kompakter Bauweise in Kälteplatten angeordnet. Ein Gerät mit einer Kälteleistung von 50 Watt und einer maximalen Temperatursenkung von $60^{\circ} \mathrm{C}$ benötigt zur Aufstellung eine Fläche von $30 \times 20 \mathrm{~cm}$, einen elektrischen Anschluß und eine Kühlwasserleitung oder einen Ventilator, um durch Luftkühlung die von der Kaltseite der Batterien abgepumpte Wärme abzuführen. Der Gleichrichter und die Siebglieder für den Gleichstrom sind in die Geräte eingebaut, ebenso ein Thermoschutzschalter, der bei Ausfall der Wasser- oder Luftkühlung den elektrischen Strom abschaltet.

In dem Standardprogramm werden Neumann-Kühlgeräte mit Kälteleistungen von 23 bis 184 Watt angeboten, die entweder eine Kälteplatte oder einen Röhrenkälteaustauscher aufweisen. Doch baut die Firma auch Sonderanfertigungen wie z. B. Kühlfallen für Vakuumvorlagen, Kühlkammern und Laborthermostate, so daß ein weites Bauprogramm von Peltierbatterien über die Kühlplatte hinaus dem Chemiker zur Verfügung steht. Diese Sonderkonstruktionen werden auch mit Kälteleistungen bis 1000 Watt angefertigt, also von Dimensionen, die bereits mit Kältekompressoranlagen in Konkurrenz treten und besonders dort eingesetzt werden können, wo eine völlig geräuschlose und wartungsfreie Kältequelle von langer Lebensdauer gefordert wird.

\title{
Inhalt
}

G. Schwen, Über die Endkonzentration von Tensiden an der Grenzfläche Lösung/Luft in Abhängigkeit von der Zeit

F. PüsCHEL, Synthese und grenzflächenaktive Eigenschaften der stellungsisomeren Natrium- und Kalium-n-hexadecylsulfate-(1) bis -(8) und einiger Natrium- und Kalium1-(n-alkyl)-n-dodecylsulfate-(1)

G. Отто, Synthetische Öle als Lederfettungsmittel
Deutsche Normen

69

Patentberichte:

Deutsche Patentanmeldungen . . . . . . . . . 86

Deutsche Patenterteilungen . . . . . . . . . . 90

Auslandspatente . . . . . . . . . . . . . . . . . 95

71 Zeitschriftenreferate . . . . . . . . . . . . . . . . 98

Buchbesprechungen . . . . . . . . . . . 105

80 Umschau . . . . . . . . . . . . 106

Der Verlag behält sich das ausschließliche Recht der Vervielfältigung in jeglicher Form, auch der der fotomechanischen Wiedergabe, vor, jedoch wird gewerblichen Unternehmen für den innerbetrieblichen Gebrauch entsprechend dem zwischen dem Börsenverein des Deutschen Buchhandels und dem Bundesversand der Deutschen Industrie abgeschlossenen Rahmenabkommen die Anfertigung einer fotomechanischen Vervielfältigung gestattet. Werden die Gebühren durch Wertmarken entrichtet, so ist eine Marke im Betrag von DM 0,30 für jedes Fotokopierblatt zu entrichten. Durch den Verlag können Fotokopien zum Preis von DM 1,60 je DIN A 4-Seite bezogen werden. Die Wiedergabe von Gebrauchsnamen, Handelsnamen, Warenbezeichnungen usw. in dieser Zeitschrift berechtigt nicht zu der Annahme, daß solche Namen ohne weiteres von jedermann benützt werden dürfen; oft handelt es sich um gesetzlich geschützte eingetragene Warenzeichen. - TENSIDE erscheint monatlich. Bezugspreis vierteljährlich DM 24,_- zuzüglich 60 Pfg. Porto. Berechnung vierteljährlich zu Quartalsbeginn. Abbestelltermin spätestens 1 Monat vor Quartalsschluß. Auslandspreis jährlich DM 98,60 einschließlich Versandkosten.

Verlag: Carl Hanser Zeitschriftenverlag GmbH (Gesellschafter Dr. Carl Hanser), München 27, Kolbergerstraße 22. Anzeigenverwaltung: Carl Hanser Zeitschriftenverlag GmbH, München 27, Kolbergerstraße 22. Gerichtsstand: München. Redaktion: Dr.-Ing. Richard E. Huttenlocher und Dr. rer. nat. Marianne Reinhardt, München, Kolbergerstraße 22, Tel. 48 5861, Telex 05-22837.

Satz, Druck und Bindearbeiten: Graphische Betriebe Dr. F. P. Datterer \& Cie. — Inhaber Sellier — Freising. Printed in Germany. 